\title{
THE X-RAY DIAGNOSIS OF ANEURYSM OF THE THORACIC AORTA.
}

\author{
By MAURICE R. J. HAYES, F.R.C.S.I.; \\ Radiologist to the Mater Misericordiæ Hospital, Dublin.
}

[Read in the Section of Medicine, March 8, 19].2.]

IN treating of the diagnosis and physical signs of aneurysm of the thoracic aorta, Professor Osler says :"The clinical picture of aneurysm of the aorta is extremely varied. Many cases present characteristic symptoms and no physical signs, while others have wellmarked physical signs and no symptoms. As Broadbent remarks, the aneurysm of physical signs springs from the ascending portion of the aorta; the aneurysm of sym. ptoms grows from the transverse arch." ("The Principles and Practice of Medicine," Fifth Edition, p. 782.'

Discussing the prognosis, he says:- "The outlook in thoracic aneurysm is always grave. Life may be prolonged for some years, but the patients are in constant jeopardy." (Ibidem, p. 783.) In dealing with this disease, therefore, it is incumbent upon us to avail of every means at our disposal to arrive at an early and accurate diagnosis. An examination of the thoracic vessels by a competent radiologist can, in the light of our present knowledge of radiology, rarely leave any doubt as to what their actual condition is. Considering the grave nature of aortic aneurysm, and how important it is to detect it as early as possible, I presume that an 
explanation of the methods of X-ray examination of the chest will be a source of interest to you.

The thorax and its contents readily lend themselves to X-ray examination, because the lungs, on account of the air which they contain, are transparent to the rays, and the heart and great thoracic vessels, being opaque, cast dense shadows.

As regards technique, in radiographing the chest more information is to be gained from the screen, or fluoroscope, than from the negative. The latter is useful only as a permanent record of the actual conditions at the time of examination, but it relatively gives very little information, and from it one can get no idea as to whether pulsation exists in the vessels. Indeed, there are some aneurysms, as I shall explain later, that can be detected by screen examination only, and, as I shall also show, there are sometimes shadows on the plate so closely simulating aneurysm that without a screen examination they might readily be labelled as such.

In order that any screen examination may be of use it is absolutely necessary that the observer should spend from five to ten minutes in total darkness before making it-this increases the sensibility of the retina-and the room in which he makes the examination should admit no light. Yet I have often seen men who have rarely, if ever, seen an X-ray tube in action, rush from bright sunlight into the X-ray room, expecting to see every detail on the screen, and when they do not see them they go away and say that the X-ray in the diagnosis of diseases of the chest is over-rated or valueless. Such an attitude is unscientific and illogical. One cannot recognise abnormalities if he does not know what is normal. It takes a large amount of practice to become conversant with the 
shadows of a normal chest, and in order to be able to give an opinion of any value one must have a large experience of the characteristics of chests that are not only normal but diseased.

The NORMAL CHEST is best examined with the patient in the erect position. (Fig. I.) With the screen on the front of the chest one sees in the middle line the shadows of the sternum and vertebral column and the great thoracic vessels; at the sides the shadows of the ribs and their interspaces-the ribs produce a lattice-like arrangement.

On the left side the heart casts a dense shadow, and its pulsations are distinctly visible. The shadow of the heart gradually merges into that of the left diaphragmatic arch during quiet respiration, but on deep inspiration the two shadows can be differentiated.

The diaphragm on the right and left sides can be clearly seen, the right arch, with the dense liver lying beneath it, being well-defined. It extends about a quarter inch higher than the left. During quiet respiration the diaphragm regularly moves upwards and downwards like a piston-the length of the stroke being from half inch to three-quarter inch, the average excursion between deep inspiration and full expiration being from two to two and a half inches. It is less in the female and in people of short stature with deep chests. Above, the clavicles and the first ribs can be seen.

The mammary glands in the female, especially when they are pendulous or well-developed, cast dense shadows which may be misleading-on moving the gland of course its shadow moves also.

With the screen on the back of the chest the same points are clearly seen, except that the heart and diaphragmatic arches, being further away from the chest 
Mr. MaURICE. R. J. Hayes_" Diagnosis of Aneurysm of the Thoracic Aorta."

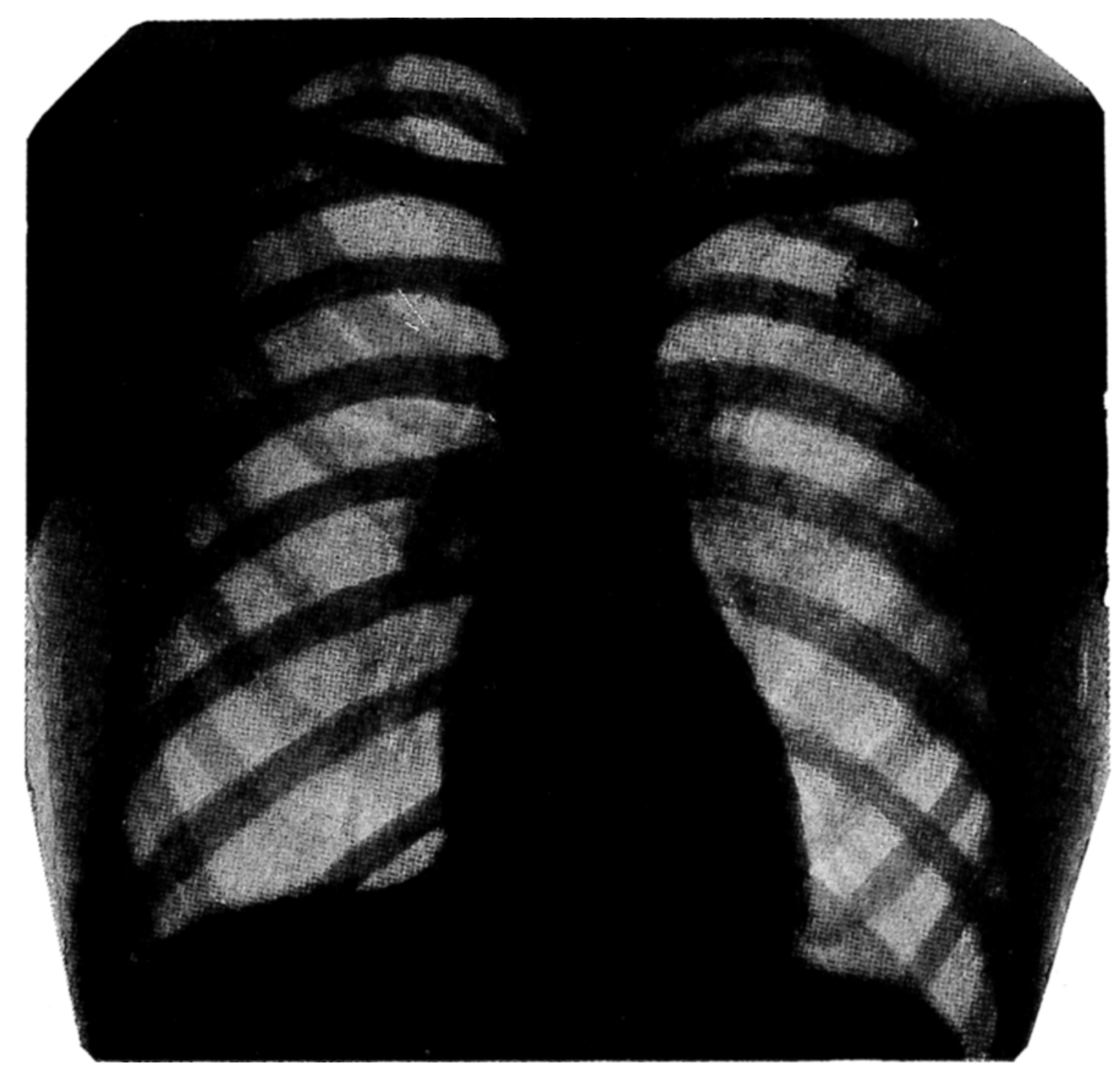

FIG. I.-Postero-anterior view of chest.

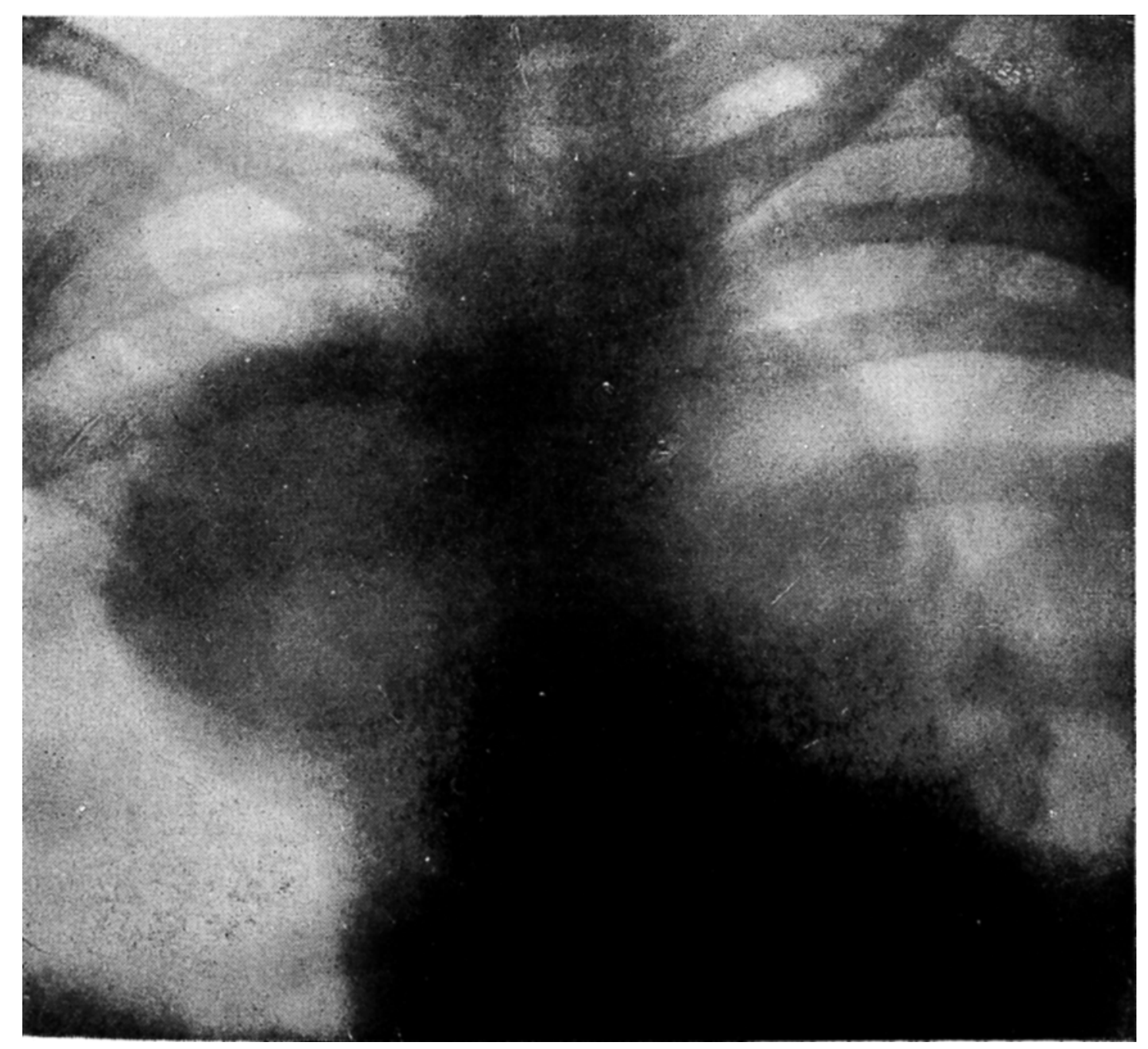

FIG. II.-A large aneurysm of the arch. It extends both to the right and to the left of the central opacity. 
wall behind, their outlines are not so well-defined. Neither is the lattice-work arrangement of the ribs seen. With the hands raised above the head, and the elbows directed forwards, the scapulæ are drawn to the sides, and do not impede our view. The lung roots can also be seen, but whether the shadows cast in this region were produced by the bronchi or by the pulmonary vessels was a point about which there was considerable controversy for many years. It is now generally agreed that these shadows are produced by the pulmonary vessels.

The Right Anterior Oblique View.-With the screen on the right side of the front of the chest, and the tube on the left side behind, an oblique view of the thorax can be obtained, and in it we can see in detail the posterior mediastinum. This is the position in which to look for aneurysms of the arch and descending aorta, and no examination of the thoracic vessels is ever complete without viewing the chest in this position.

In the left posterior oblique position, with the screen on the left side of the chest behind, and the tube on the right side of the chest in front, the entire intra-thoracic portion of the descending aorta can be studied. This position is also the best for examination of the thoracic portion of the csophagus for stricture.

In the left lateral position, with the screen on the left side of the chest and the tube on the right side, the antero-posterior diameter of the heart can be determined. In front of the heart is a clear space called the retrosternal triangle. It is formed by the sternum in front, the diaphragm below, and the anterior border of the heart posteriorly. Behind the heart is another clear triangular space called the retro-cardiac triangle. It is formed by the vertebral column behind, by the diaphragm 
below, and by the posterior margin of the heart in front; and here one can plainly see bismuth food pass into the stomach, or an aneurysm of the descending aorta if present. In most healthy adults, more especially those living in cities, some enlarged glands around the root of the lung and in the posterior mediastinum can be seen. Such are the radioscopic appearances of the normal chest.

It is with the screen and not from the plate that the diagnosis of aneurysm must be made, and the chest must be carefully examined from the front and from the back, as well as in the right anterior oblique and the left posterior oblique positions.

In the anterior and posterior examinations, as already explained, the shadows of the great thoracic vessels are in line with those of the sternum and vertebral column. A little to the right of the spine is seen a less dense shadow produced by the superior and inferior venæ cavæ.

That part of the aorta formed by the junction of its transverse and descending portions, called by Walsham and Orton "the left lateral aortic bulge," is not very prominent in young people, and it is always less evident in females than in males. Its size and situation must, however, be carefully noted, as it is a common source of error in the interpretation of negatives. If aneurysms are present they will appear as dark shadows to the right or left of the central dark area. They may or may not be pulsating, and their margins will be defined in proportion to the amount of clot which they contain.

Aneurysms of the aorta can, as a rule, be recognised without much difficulty, but it is not always easy to detect those arising from the innominate, the left common carotid, or the left subclavian arteries, because of the 
Mr. Maurice R. J. Hayes-" Diagnosis of Aneurysm of the Thoracic Aorta."

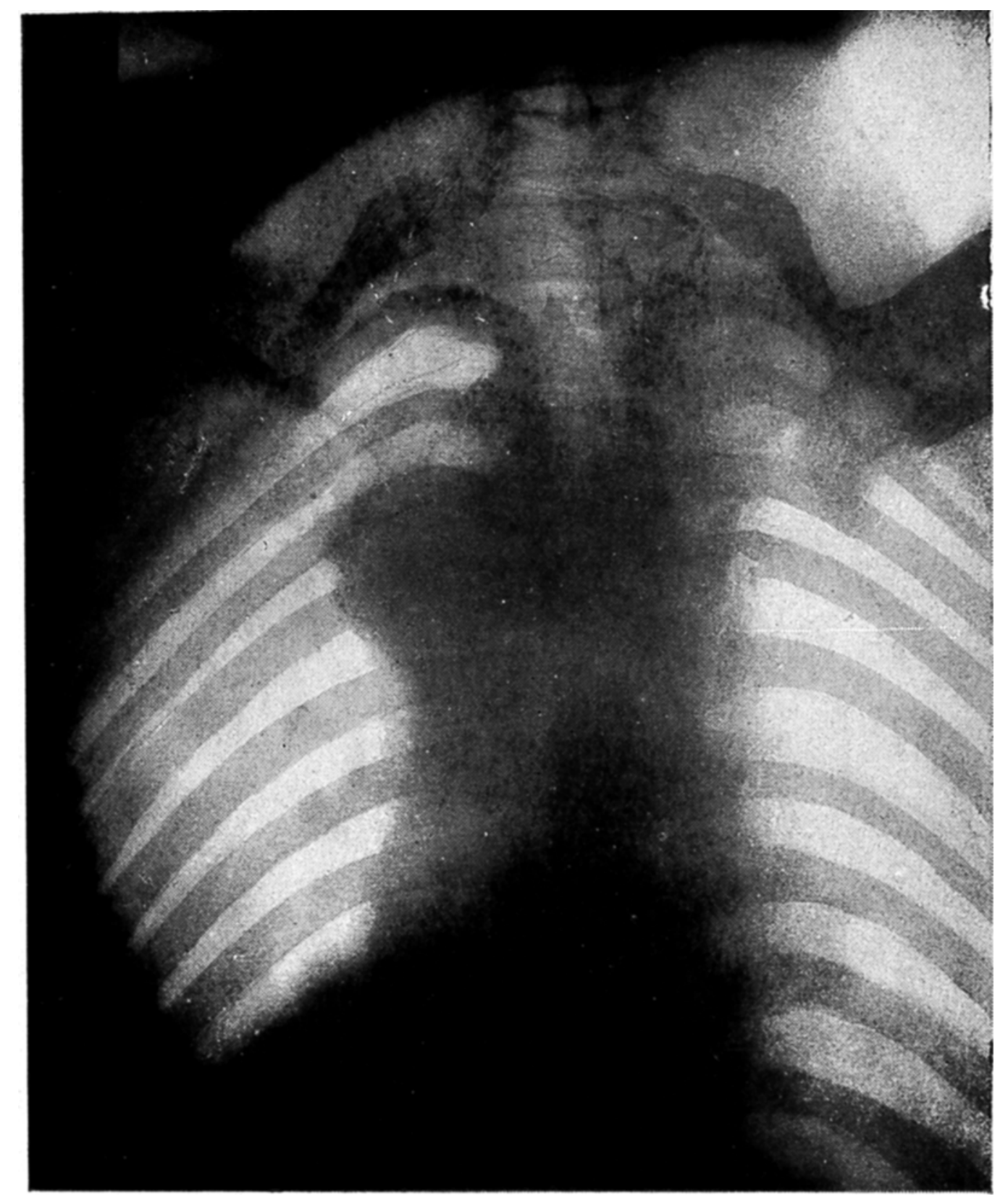

FIG. III.-Small aneurysm at junction of transverse and descending portions of arch. The chest is viewed from behind.

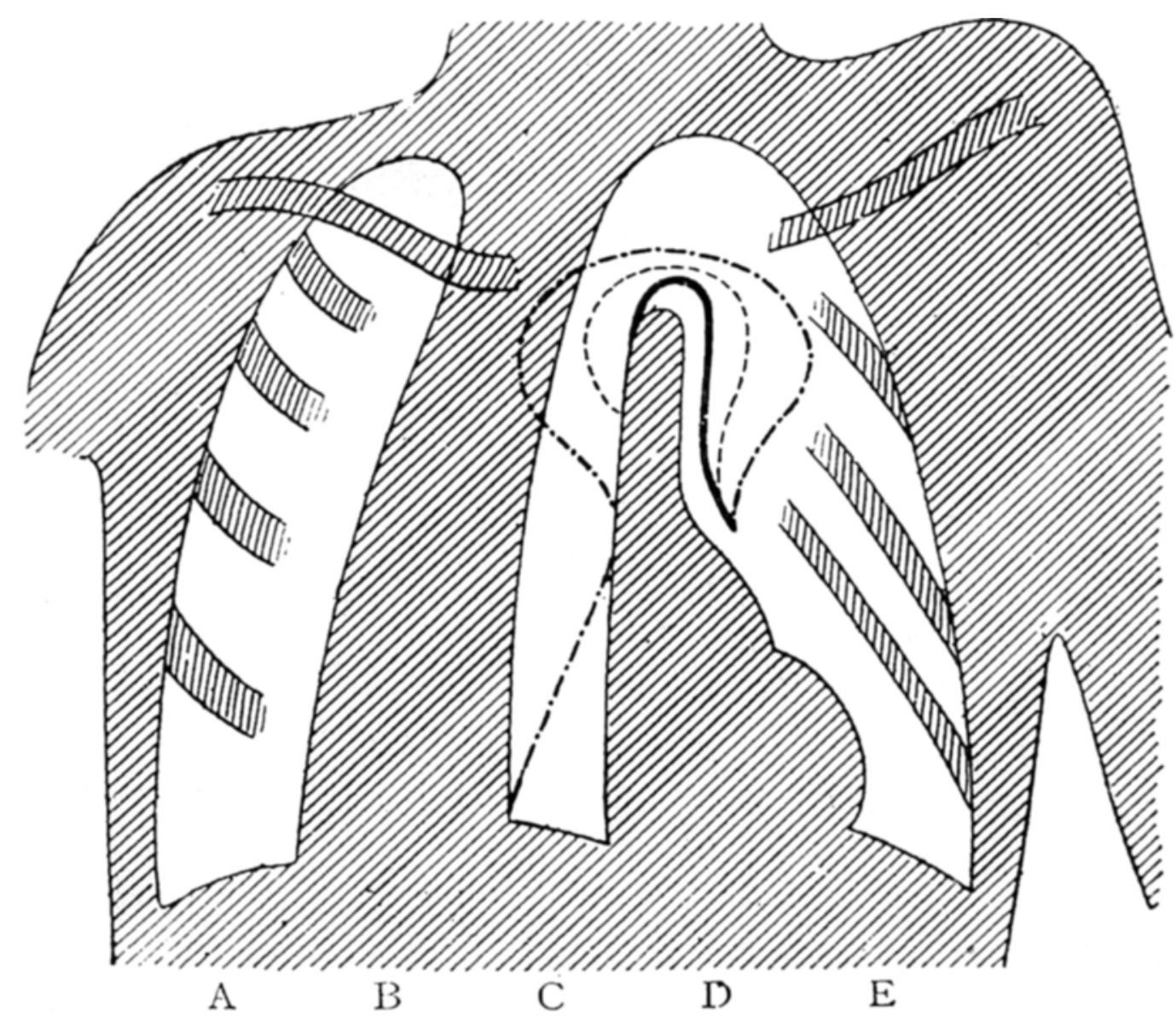

Fig. IV.-Rigu't Anterior Oblique (aFter Holzknecht.) (From "Röntgen Rays in Chest Disease." By Walsham and Orton.) Dilated aorta. Small commencing aneurysm.

-.-.- Upper part, larger aneurysm; lower part, dilated auricle. 
converging upper limits of the thoracic cavity and the smaller amount of transparent lung tissue here situate.

Aneurysms to the right of the vertebral column are, as a rule, on the ascending aorta, those to the left on the descending aorta. A large aneurysm of the arch of the aorta (Fig. II.) may extend both to the right and to the left of the central opacity formed by the sternum and vertebral column, or it may extend upwards towards the root of the neck. Small aneurysms of the transverse and descending portions of the arch will cast shadows to the left of the vertebral column. (Fig. III.) The lateral and oblique positions will help to determine the depth at which an aneurysm is situate. The aneurysm is also nearer to that surface of the chest on which, when viewing it, its limits are most clearly defined.

To give an accurate diagnosis of aneurysm the left anterior oblique view should never be orritted, and a detailed description of the shadows as they appear on the screen when viewed in this position must be understood. In this view (Fig. IV.) the shadow cast by the heart and great vessels is not at all unlike that produced by the closed hand with the index finger extended (D), the fist corresponding to the heart, and the index finger to the ascending transverse and descending portions of the aorta. To the right of this is the patient's left lung $(\mathrm{E})$, and to the left an elongated clear space, in which lies the œsophagus (C); next the dark shadow of the vertebral column is seen (B), and outside it, on the observer's left side, is a clear space corresponding to the patient's right lung (A).

The shadow corresponding to the index finger has parallel sides and a rounded upper end.

In aneurysms of the arch this upper end assumes a 
dumb-bell appearance, having a rounded head and a neck. A generally dilated aorta, due to atheroma, may give rise to symptoms simulating aneurysm, and its radiographic, appearances in an antero-posterior view will very closely simulate one. The diagnosis of this condition can be accurately made only by examining the chest in the oblique position, when the sides of the index finger shadow will be seen to be parallel, and its upper end will not be bulbous.

From an examination of the chests of a large number of cases, some of whom suffered from angina without any apparent cause for their pain, and others, who were thought to have aneurysm, but in whom it did not exist; and many whom I have examined for stricture of the cesophagus, I must say that general dilatation of the aorta is more common than it is supposed to be. It is the rule to find this condition in most men beyond the age of fifty who have led strenuous lives, although there may be associated with it no evidence of the existence of atheroma in the peripheral vessels. It would be of interest to know if the findings in the post-mortem room support this view.

Other shadows occurring within the thorax, which may be mistaken for aneurysm, may be produced by :-

1. New growths.

2. Consolidation of the lung.

3. Abscess or empyema.

4. Enlarged glands.

1. The shadows of new growths are denser than those of aneurysms, their borders are indistinct and ill-defined, and they are not expansile. (Fig. V.) That they may be effected by transmitted pulsation must be borne in mind.

2. A focu's of consolidation in the lung, if small and 
Mir. Maurice R. J. Hayes-"Diagnosis of Aneurysm of the Thoracic Aorta."

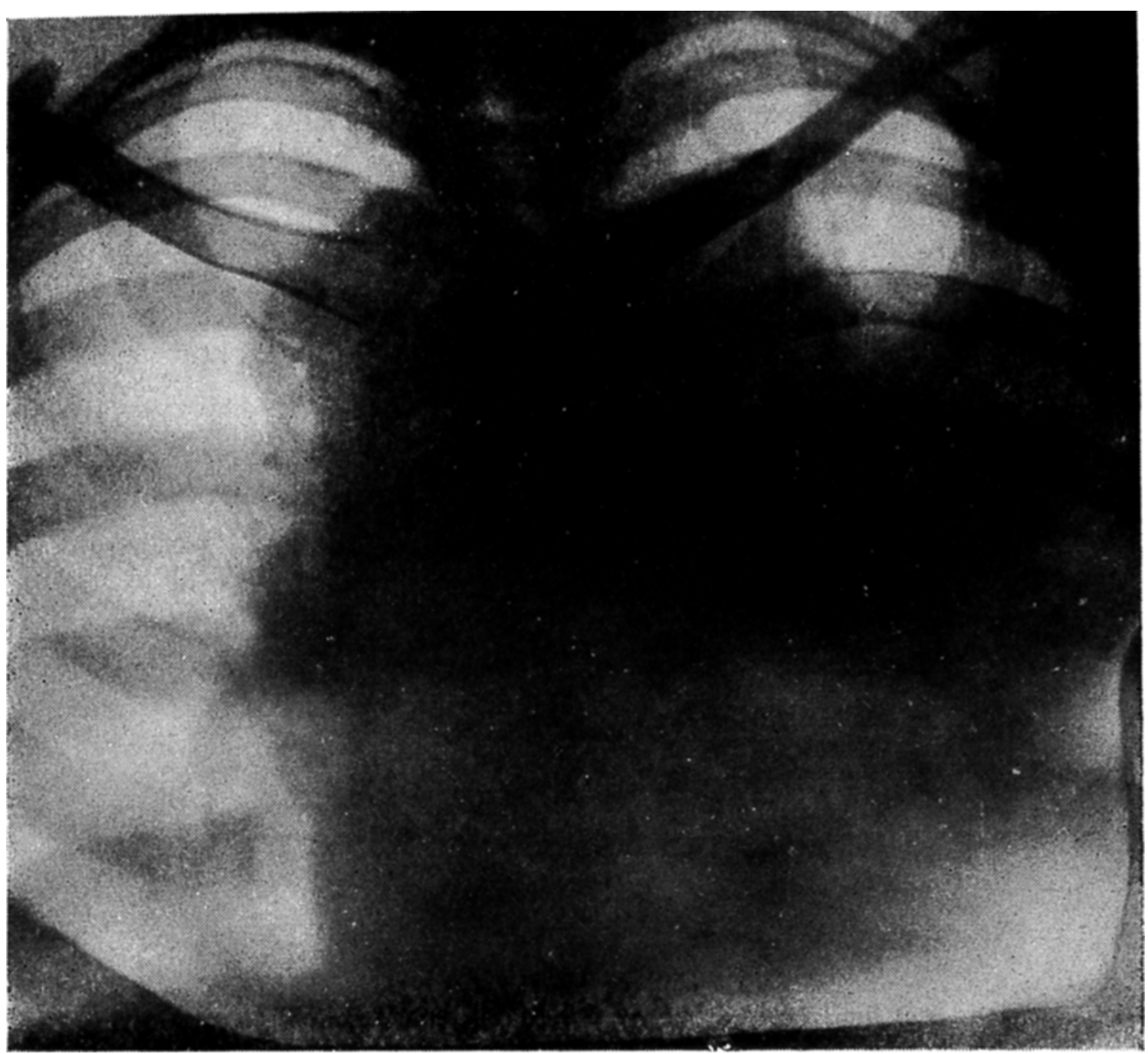

Fig. V.-Lympho-sarcoma.

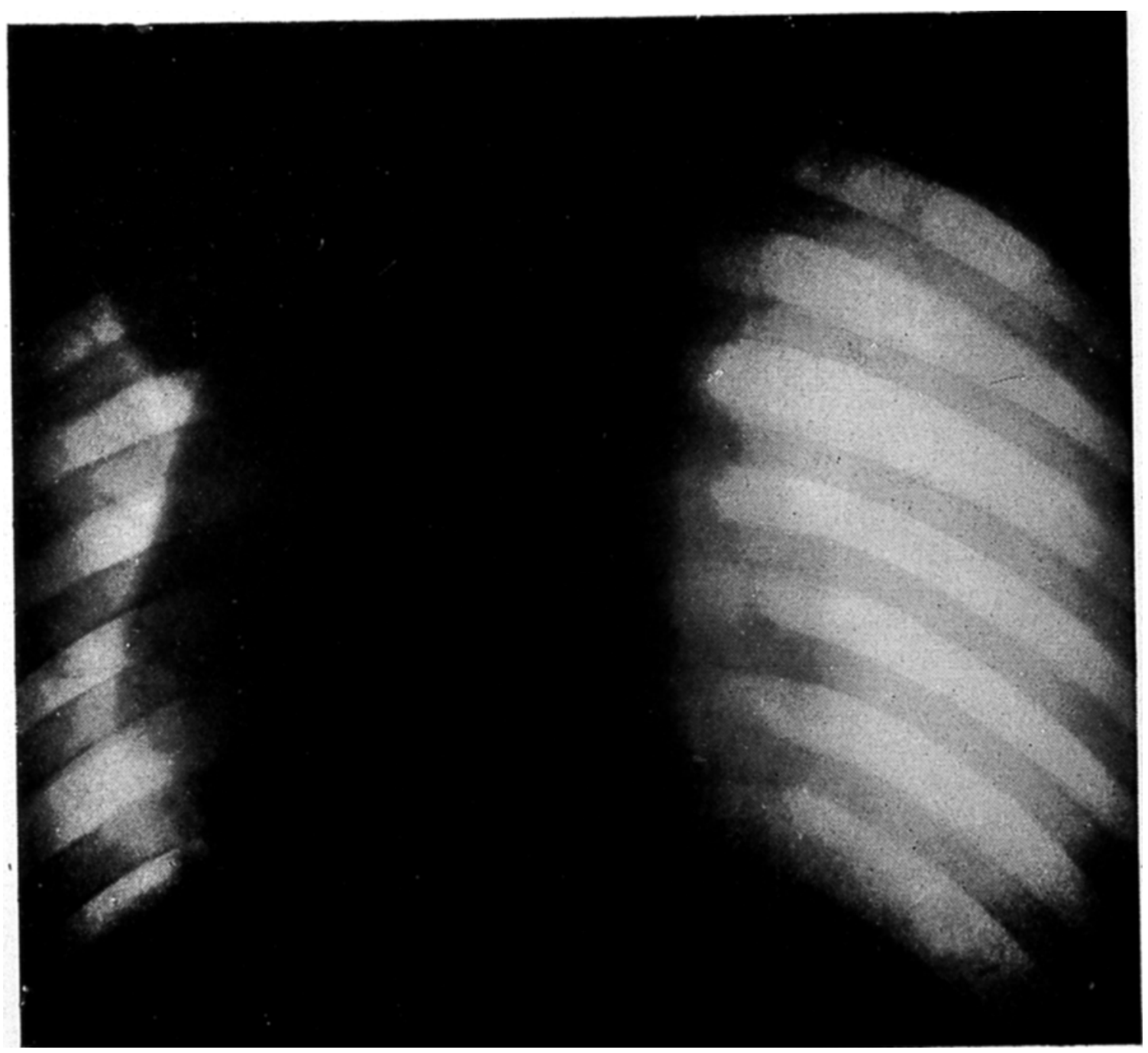

TIG. VI.-Aneurysm of the arch of the aorta with aneurysm of the left common carotid antery. 
centrally situated, will be entirely surrounded by the transparent lung tissue, and it will be independent of the great vessels. Expansile pulsation must also be sought for, and the outline of the shadow carefully noted. At the root of the neck it is extremely difficult to differentiate between aneurysms arising from the branches of the arch of the aorta and consolidation of the lung apex (Fig. VI.), and in arriving at a diagnosis the antecedent history of the patient, as well as the symptoms and clinical signs present, must be taken into account.

3. Abscesses or empyema will be recognised by their situation, their shape, and the history.

4. Enlarged glands produce scattered shadows to either side of the vertebral column, and their appearances are so typical that they are not likely to cause any ambiguity.

Another point to be observed in cases of aneurysm is that the long axis of the heart, instead of being oblique, becomes almost horizontal, the base of the heart being depressed and the apex elevated, and this position is independent of any pushing up of the left diaphragm through flatulent distention of the stomach or any such cause.

The position of the heart varies with the position of the patient, but within narrow limits, and the degree of lateral displacement of this organ on rotating the patient from side to side when in the recumbent position is rarely more than one inch in either direction. One case has been recorded in which the entire heart could be displaced for several inches to the right or left by rotating the patient (Silbergleit, Medical Record, June 20th, 1903). I have seen a freeiy movable heart in two patients, both of whom were elderly men. In each of these cases, on examining the patient in the erect position, the heart 
94 Diagnosis of Aneurysm of the Thoracic Aorta.

swayed pendulum-like from side to side, and the swinging movements of the heart were synchronous with the ventricular contractions, and they appeared to be determined by them. They were independent of respiration. One of these patients had an aneurysm of the descending aorta just above the diaphragm-a slide of this case I shall show you. The other had a dilated aorta, but no aneurysm. In each case, however, the heart was pulsating rapidly but regularly. This swinging movement of the heart was not seen on examining these patients in the recumbent position. It is a phenomenon which I have not seen described, and I can suggest no other explanation of it than that it was possibly due to an elongated and abnormally free ascending aorta. 